\title{
Respuestas ambivalentes ante la moralidad ambigua del antihéroe: Tony Soprano y Walter White como casos de estudio*
}

\section{Fernando Canet $^{1}$ Alberto Nahum García-Martínez²}

Recibido: 2017-03-06

Enviado a pares: 2017-03-16
Aprobado por pares: 2017-05-16

Aceptado: 2017-06-06

DOI: $10.5294 /$ pacla.2018.21.2.5

Para citar este artículo / to reference this article / para citar este artigo Canet, F. y García-Martínez, A. N. (2018). Respuestas ambivalentes ante la moralidad ambigua del antihéroe: Tony Soprano y Walter White como casos de estudio. Palabra Clave, 21(2), 364-386. DOI: 10.5294/pacla.2018.21.2.5

\section{Resumen}

La popularización del antihéroe va anudada a la necesidad dramática y narrativa de la serialidad: la constante dicotomía entre esfera familiar y profesional, entre acciones nobles y mezquinas, entre deber y querer, permite hacer avanzar la trama, generar suspenso y renovar los conflictos, lo que hace que el relato se dilate decenas de horas. Vic Mackey (The Shield), Dexter Morgan (Dexter) o Nucky Thompson (Boardwalk Empire) podrían servir de ejemplo antiheroico. Pero, sin duda, los dos casos más radicales y complejos son los protagonistas de Los Soprano (Tony Soprano) y Breaking Bad (Walter White). La identificación emocional con el antihéroe ha empezado a estudiarse recientemente. Este artículo propone un nuevo ángulo de estudio: la relación del antihéroe con el resto de personajes de la serie. Para ello, seleccionaremos los caracteres — principales o secundarios-que

\footnotetext{
* Advertimos spoilers: este artículo describe escenas críticas e importantes para la trama de las series The Soprano y Breaking Bad, con el fin único de ejemplificar los objetivos y conclusiones que desarrollan los autores durante el texto.

1 orcid.org/0000-0002-1455-9249. Universidad Politécnica de Valencia, España. fercacen@upv.es

2 orcid.org/0000-0002-4017-5791. Universidad de Navarra, España. albgarcia@unav.es
} 
los guionistas emplean como estrategia narrativa para guiar las respuestas emocionales y morales del espectador en su particular relación con el antihéroe. Principalmente, nos centraremos en las reacciones que estos personajes exhiben hacia las conductas amorales de los antihéroes. Proponemos una metodología de análisis textual que, partiendo de teóricos cognitivistas, nos servirá como mecanismo para entender mejor la construcción del antihéroe e indagar sobre los efectos que las decisiones autorales provocan en los espectadores.

\section{Palabras clave}

The Sopranos; Breaking Bad; teoría cognitiva; estudios de televisión; vínculos con los personajes; narrativas del antihéroe; emociones morales (Fuente: Tesauro de la Unesco). 


\section{Ambivalent Responses to the Ambiguous Antihero Morality: Tony Soprano and Walter White as a Case Study*}

\section{Abstract}

The popularization of the antihero is linked to the dramatic and narrative need of seriality: the constant dichotomy between the family and professional spheres, between noble and petty actions, desire, moves the story forward, generates suspense and renews conflicts. This prolongs the story for dozens of hours. Vic Mackey (The Shield), Dexter Morgan (Dexter) or Nucky Thompson (Boardwalk Empire) could serve as an antihero example. However, the two most radical and complex cases are undoubtedly the protagonists of The Sopranos (Tony Soprano) and Breaking Bad (Walter White). The emotional affinity with the antihero has recently become the object of study. This paper proposes a new angle of study: the antihero's relationship with the rest of the characters in the series. For that purpose, we will select the characters - main or secondary - that the writers use as narrative strategy to guide the emotional and moral responses of the spectator the emotional and moral responses of the viewer in their particular relationship with the antihero. We will mainly focus on the reactions of these characters to the amoral behaviors of the antiheroes. We propose a methodology of textual analysis that, based on cognitivist theorists, will serve as a mechanism to better understand the construction of the antihero and to investigate the effects of authorial decisions on viewers.

\section{Keywords}

The Sopranos; Breaking Bad; cognitive theory; television studies; character engagement; antihero narratives; moral emotions (Source: Unesco Thesaurus).

* Disclaimer: This article describes critical and important scenes about the series The Soprano and Breaking Bad. They are used as examples that help the authors develop their objectives and conclusions through the work. 


\section{Respostas ambivalentes perante a moralidade ambígua do anti-herói: Tony Soprano e Walter White como casos de estudo*}

\section{Resumo}

A popularização do anti-herói vai ligada à necessidade dramática e narrativa da serialidade: a constante dicotomia entre esfera familiar e profissional, entre ações nobres e mesquinhas, entre dever e querer, permite o avanço da trama, geração de suspenso e renovação dos conflitos, dilatando o relato durante dezenas de horas. Vic Mackey (The Shield), Dexter Morgan (Dexter) ou Nucky Thompson (Boardwalk Empire) poderiam servir como exemplo anti-heróico. Mas, sem dúvida, os dois casos mais radicais e complexos são os protagonistas de Familia Soprano (Tony Soprano) e Breaking Bad (Walter White). A identificação emocional com o anti-herói começou a ser estudada recentemente. Este artigo propõe um novo ângulo de estudo: a relação do anti-herói com o resto de personagens da série. Para isso, selecionaremos os caracteres - principais ou secundários - que os roteiristas empregam como estratégia narrativa para guiar as respostas emocionais e morais do espectador em sua particular relação com o anti-herói. Principalmente, nos concentraremos nas reações que estes personagens exibem com relação às condutas amorais dos anti-heróis. Propomos uma metodologia de análise textual que, partindo de teóricos cognitivistas, nos servirá como mecanismo para entender melhor a construção do anti-herói e indagar sobre os efeitos que as decisões autorais provocam nos espectadores.

\section{Palavras-chave}

Los Soprano; Breaking Bad; teoria cognitiva; estúdios de televisão; envolvimento com os personagens; narrativas dos anti-heróis; emoções morais (Fonte: Tesauro da Unesco).

\footnotetext{
* Alerta de spoiler: este artigo descreve cenas críticas e importantes da série Família Soprano e Breaking Bad, com o único propósito de exemplificar os objetivos e conclusões desenvolvidos pelos autores durante o texto.
} 


\section{Introducción}

La popularidad de las series televisivas es un hecho conocido y aceptado por todos los agentes que intervienen en producción, consumo, análisis y crítica del audiovisual contemporáneo: profesionales, audiencia, críticos y académicos. Una de las características que define este boom es la complejidad estética y moral de sus propuestas. Jason Mittell en su reciente libro Complex TV: The poetics of contemporary television storytelling (2015) ha sido uno de los primeros académicos que ha intentado acotar y definir este fenómeno. Para este autor norteamericano, el rol de los personajes es vital en la complejidad de esta nueva serialidad. En su libro, dedica especial interés a cómo estos son caracterizados y cómo este acto creativo motiva en el espectador un compromiso emocional con aquellos (Mittell, 2015, p. 118). Así, la complejidad de la serialidad contemporánea requiere personajes igualmente problemáticos como uno de los principales mecanismos para garantizar la fidelidad del espectador a lo largo de las diferentes temporadas.

Un ejemplo de este tipo de personajes es la figura del antihéroe. Su complejidad explica el amplio número de series recientes que explotan su protagonismo de una forma muy sugestiva. ${ }^{3}$ Los Soprano (The Sopranos, David Chase, HBO, 1999-2007), Dexter (James Manos Jr, Showtime, 2006-2013), Breaking Bad (Vince Gilligan, AMC, 2008-2013), Boardwalk Empire (Terence Winter, HBO, 2010-2014) y Peaky Blinders (Steven Knight, BBC Two, 2013) son un ejemplo de ello. Uno de los motivos de la complejidad de estos personajes es su ambigüedad moral, que busca provocar en el espectador respuestas contradictorias. A diferencia de la noción de antihéroe canónica hace unas décadas (Frye, 2006, p. 151), en la actualidad se refiere a un personaje que toma prestados aspectos tanto del héroe como del villano (García, 2016, p. 53; Vaage, 2016, p. 93). Mientras que el héroe clásico lleno de virtudes y el villano repleto de vicios invitan al espectador a aceptar al primero y a rechazar al segundo sin apenas vacilación, el antihéroe enigmático, por el contrario, invita a un tipo de relación que se torna moralmente enrevesada para el espectador.

3 Aquellos interesados en explorar las razones de esta pujante irrupción pueden leer la sección titulada “The emergence of antiheroism in contemporary TV” en Emotions in contemporary TV series (García, 2016, pp. 53-55). 
El principal promotor de esta tendencia en el panorama actual ha sido, sin duda, Los Soprano, digna heredera de las películas de gánster de la época dorada del clasicismo, ${ }^{4}$ pero, sobre todo, de su reescritura posterior en la trilogía de El Padrino (The Godfather, The Godfather II, The Godfather III, Francis Ford Coppola, 1971, 1973, 1990). En esta saga, Coppola asienta las bases del tratamiento actual del antihéroe en las series televisivas mediante su decisión de tratarlo como el protagonista a través del cual narrar la historia. Así, la prevalencia del punto de vista del antihéroe permite su acercamiento al espectador conllevando que este conozca, entre otras cosas, las razones de sus actos. Un año después de la finalización de Los Soprano nace otro de los ejemplos emblemáticos de la figura del antihéroe. En Breaking Bad, asistimos a la transformación de Walter White, un hombre ordinario, en Heisenberg, uno de los antihéroes más fascinantes de este boom televisivo.

Debido a la relevancia de estas dos series, sus respectivos protagonistas van a ser objeto de análisis en este artículo. Su estudio nos permitirá explorar cómo las series actuales llevan a cabo el tratamiento de esta figura tan controvertida. Aunque se trata de un tema muy reciente, ya existe una literatura académica relevante que lo aborda. Hay artículos, capítulos y libros publicados al respecto, principalmente sobre la figura de Tony Soprano, (Lavery, 2002, 2006; Carroll, 2004; Polan, 2009; Smith, 2011; Toscano, 2014), pero también acerca de Walter White (Hernández, 2013; Wondemaghen, 2015; García, 2016), así como, incluso, bibliografía que aborda los dos ejemplos como caso de estudio (Rush, 2014; La Berge, 2015). Además, hay que destacar el reciente libro escrito por Margrethe Bruun Vaage, The antihero in American television (2016), que, como el título describe, versa específicamente acerca de la figura del antihéroe. Aunque Vaage hace alusión a otros protagonistas, la relevancia tanto de Tony Soprano como de Walter White es más que significativa a lo largo de todo el volumen.

4 Nochimson (2002) propone una relectura del género de los gánsteres a través de Los Soprano en su artículo "Waddaya lookin' at?". 
Nuestra intención en este artículo es hacernos acopio de esta literatura para entender mejor cómo es tratado el antihéroe en la serialidad contemporánea como tendencia representativa. Ahora bien, lo queremos hacer desde una perspectiva metodológica diferente. Nuestra propuesta es alcanzar este objetivo a través del análisis de cómo es trazada la relación del antihéroe con el resto de los personajes a lo largo de la serie. Para ello, seleccionaremos aquellos que desde nuestro punto de vista son utilizados por los creadores como estrategia narrativa, en su intención de guiar las respuestas emocionales y morales del espectador en su particular relación con el antihéroe. Principalmente, nos centraremos en las reacciones que estos personajes tienen hacia las conductas amorales de sus respectivos antihéroes. Así pues, proponemos una metodología de análisis textual que nos servirá de mecanismo no solo para entender mejor la construcción del antihéroe, sino también para indagar sobre los efectos que las decisiones autorales puedan provocar en los espectadores. Estos objetivos se llevarán a cabo a continuación a través de dos secciones: una dedicada al análisis de Los Soprano y la otra al de Breaking Bad, cerrando el artículo un apartado en el que se ofrezcan conclusiones que sirvan para satisfacer los propósitos planteados.

\section{Los Soprano: complicidades, moralidades y amenazas}

La serie de David Chase resulta un ejemplo excepcional para llevar a cabo nuestro objetivo, ya que en ella encontramos una representación significativa de las diferentes posiciones que se pueden mantener hacia la figura del antihéroe. Así, a través de una amalgama de personajes, y de cómo estos se relacionan con Tony Soprano, los espectadores pueden encontrar una diversidad de espejos a través de los cuales pueden reflejarse en una hipotética relación con este protagonista contradictorio. Algunas de estas relaciones son estáticas, pero otras evolucionan conforme lo hace la serie. En primer lugar, nos centraremos brevemente en las primeras, que implican una relación más superficial con el antihéroe, para, posteriormente, abordar las segundas que por su nivel de profundidad y evolución resultan centrales a fin de entender la complejidad moral que los autores de la serie proponen a sus espectadores. 
En el primer grupo, incluimos aquellos secundarios que mantienen una relación puntual y superficial con Tony Soprano. Se trata de personajes que no pertenecen a su mundo y que lo observan y evalúan desde la distancia, ofreciendo definiciones estereotipadas, rasas y polarizadas de este. Por otra parte, tales personajes tienen en común que, de una forma u otra - como colega, amigo, amante o exmarido — están vinculados con la psiquiatra, Dra. Jennifer Melfi (quien, como veremos más abajo, forma parte del segundo grupo propuesto). En muchos casos (por ejemplo, en el del exmarido de Melfi), la función es persuadirla a que no se vincule profesionalmente con Tony. A esta misma tarea se aplica, de forma más permanente, su terapeuta, el Dr. Elliot Kupferberg, que intentará por todos los medios convencer a Melfi de la inutilidad de la terapia con pacientes como Soprano. En estos personajes, la posición de rechazo es incondicional hacia la figura de Tony. Ellos destacan en sus valoraciones solo el lado más oscuro del mafioso para crear antipatía hacia él. Por otro lado, la reacción de estos personajes hacia Tony también se puede definir respecto de la curiosidad y fascinación que este personaje, y su mundo, tiene de excepcional; así como el respeto y miedo por las posibles consecuencias de verse envueltos en su violenta vida. Precisamente, los diferentes teóricos destacan lo seductor, atractivo y poderoso que puede llegar a ser el antihéroe (Plourde, 2006, p. 75; Carroll, 2004, p. 124; Smith, 2011, p. 83; Mittel, 2015, p. 157; Vaage, 2016, pp. 98, 107).

Por lo que respecta al segundo grupo (aquellos que mantienen una relación central a lo largo de toda la serie con Tony), nos enfocaremos en tres que, por diferentes motivos que ahora veremos, son los más representativos: su mujer, Carmela Soprano, su sobrino, Christopher Moltisanti, y su terapeuta, la ya citada Dra. Melfi. Los dos primeros forman parte de su familia, mientras que la tercera es ajena a esta vinculación tan importante en este tipo de propuestas genéricas. Ahora bien, a su vez, existe una diferencia relevante entre Carmela y Christopher en su relación con Tony. Mientras la primera representa, junto con sus dos hijos, esa parte de la familia que Tony quiere mantener alejada de cualquier vinculación con el lado oscuro, el segundo se verá obligado por este a saborear la parte más amarga de esta oscuridad. Estos tres personajes — con sus matices, como detalla- 
remos - son aquellos que los autores principalmente utilizan en la serie para cuestionarles a los espectadores su "proactitud" o simpatía hacia Tony.

La razón principal es que los tres, por su condición, no merecen sufrir las consecuencias negativas de verse envueltos en la vida de un personaje como Tony Soprano. A diferencia de quien sí se lo merece (aquellos villanos sin escrúpulos que irán apareciendo a lo largo de la serie), estos personajes no merecen ni sufrir la violencia directa de Tony ni tampoco las consecuencias colaterales de su relación con él. Los diferentes teóricos han destacado que uno de los factores clave a la hora de justificar moralmente la violencia del antihéroe es precisamente el nivel moral de quien la sufre (Carroll, 2004, p. 131; Mittel, 2015, p. 146; Vaage, 2016, p. 103). Es decir, si la víctima de esta se lo merece, la justificación del acto violento será más probable por parte del espectador. En el episodio "Big Girls Don't Cry” (2x05, Tim Van Patten, HBO, 2000), el propio Tony se autojustifica diciéndole a la Dra. Melfi que él dirige su poder y su ira en contra de quien se lo merece. En este sentido, el espectador lo reconoce como un justiciero que, aunque fuera de la ley, actúa en la dirección correcta y así absuelve moralmente sus conductas delictivas, ya que el fin de ellas es valorado como adecuado.

Incluso en algunos casos los autores articulan estrategias que invitan al espectador no solo a justificarlas sino también a reclamarlas para restablecer la violada justicia. Un ejemplo de ello lo podemos ver en el capítulo "Employee of the Month" (3x04, John Patterson, HBO, 2001), en el que la Dra. Melfi es violada y, además por la incompetencia del sistema judicial, su agresor de forma incomprensible queda libre de toda culpa. Como muy bien apunta Vaage, estas circunstancias provocan que el espectador quiera que Melfi se lo cuente a Tony para que él haga uso de su potestad y se vengue en su nombre (Vaage, 2016, p. 131). Finalmente, aunque con gran tentación, la Dra. Melfi no lo hace. Las posibles consecuencias pesan más que su apetito de venganza y así frustra los deseos de los espectadores que, cómodamente instalados fuera de la ficción, no tienen en cuenta en su valoración, a diferencia de la psiquiatra, las consecuencias del deseo vengativo. Si nos acercamos a las opiniones que los usuarios de IMDb han hecho de este capítulo, veremos cómo su objetivo, plantear un dilema moral, se 
ha cumplido satisfactoriamente. Uno de ellos dice: "We WANTED Tony to commit murder and it wouldn't have seemed wrong if he had" [Nosotros QUERÍAMOS que Tony cometiera el asesinato y no hubiera parecido erróneo si lo hubiera hecho]. Y otro: "She went the right way even though most of our society love to act like an angry mob" [Ella escogió el camino adecuado a pesar de que a la mayoría de nuestra sociedad le encanta actuar como una muchedumbre enfadada].

Ahora bien, ¿cuál sería la respuesta de los espectadores en el caso de que quien sufriera en sus propias carnes la violencia de Tony fuese su sobrino Christopher? Antes de explorar las posibles respuestas, debemos situar al lector. Como ya se narra en el primer episodio, Christopher es una víctima de las voluntades de Tony. Mientras que aquel se quiere abrir camino fuera de la ilegalidad, este le tiene previsto otros caminos. Tony quiere que su sobrino deje sus sueños cinematográficos ("Forget Hollywood screenplays" [Olvídate de los guiones de Hollywood]) y se centre en los negocios ilegales de la familia ("We got work to do. New avenues" [Tenemos trabajo que hacer. Nuevos caminos]). Así, el responsable de que Christopher cruce la línea, se instale en el terreno de la inmoralidad y acabe en desgracia es, principalmente, su tío. Por otro lado, su obligado viaje por la senda de la ilegalidad ha llegado a tal extremo que su ocaso implica una amenaza permanente para la continuidad de Tony. En cualquier momento aquel lo puede delatar o sus acciones indisciplinadas pueden poner en peligro al jefe del clan Soprano.

Ahora sí el dilema está servido: ¿el espectador justificará en este caso el hecho de que Tony acabe con la vida de su propio sobrino? Planteando esta disyuntiva moral, los autores están llevando al límite el compromiso que el espectador tiene con quien ha sido su protagonista a lo largo de seis temporadas. Uno de los principales argumentos que García desarrolla en su capítulo "Moral emotions, antiheroes and the limits of allegiance" (2016, pp. 52-70) es el hecho de que la narración articula estrategias cíclicas con la intención de cuestionar la lealtad del espectador hacia el antihéroe, a pesar de sus conductas inmorales. Para ello, incluso, se construyen situaciones extremas donde este cuestionamiento se lleva al límite. Un 
ejemplo de ello sería este que estamos viendo, desarrollado en el capítulo "Kennedy and Heidi" (6x18, Alan Taylor, HBO, 2007). Aprovechando un accidente de tráfico, Tony asesina a su sobrino y así, como él mismo admite, "the biggest blunder of [his] career is now gone" [la mayor metedura de pata de su carrera se ha terminado]. ¿ Justificarán los espectadores este exceso de inmoralidad? En los casos en que lo hagan, que podemos pensar que será en su gran mayoría, así lo harán como consecuencia del trabajo que la narración ha llevado a cabo a lo largo de la serie, forzando la degradación de Christopher. Hasta tal extremo que el espectador considera, como así lo hace su tío, que su muerte es merecida, aun a pesar de que sea él el responsable inicial del viaje al lado oscuro de su sobrino. Ahora bien, no solo Christopher se merece desaparecer por su degradación moral en sí misma, sino porque esta se está convirtiendo en la primordial amenaza para la continuidad de nuestro protagonista, esto es, en el principal obstáculo para sus logros. Y si la narrativa nos ha enseñado algo desde sus ancestros, es que lo que más desea el espectador es que su protagonista alcance sus propósitos al final de su viaje. Incluso en aquellos casos en los que su moralidad es puesta en controversia tenazmente a través de su relación con otros personajes, como así sucede en los casos de la figura de los antihéroes televisivos contemporáneos.

A nuestro entender, Carmela y la Dra. Melfi son las dos caras de una misma moneda. Ambos personajes se ven en la tesitura de tomar una decisión ante las pretensiones de Tony. Ahora bien, mientras que la respuesta de Carmela es afirmativa, la de la Dra. Melfi es todo lo contrario. Carmela, a pesar de conocer su naturaleza delictiva, aceptó casarse con él. Carmela se lo reconoce en terapia a la Dra. Melfi cuando, en "Mayham" (6x03, Jack Bender, HBO, 2006), le confiesa: "I don't know if I loved him in spite of it or because of it” [No sé si lo amaba a pesar de o a causa de]. Así, Carmela, de forma consciente, decidió cruzar la línea motivada solo por las ventajas que suponía verse involucrada en la vida de un personaje como Tony y sin tener en cuenta lo más mínimo las desventajas de hacerlo. Precisamente, el temor a las consecuencias negativas de su toma de decisión y sentirse cómplice de las atrocidades que su esposo comete son los principales motivos de los conflictos que Carmela evidencia a lo largo de la serie. Carmela es 
caracterizada como una mujer católica que, como tal, vive con amargura su perpetua contradicción. Para suavizar tal discordancia, busca obsesivamente razones que acentúen la cara más amable de su esposo. Así, por ejemplo, durante su terapia con el Dr. Krakower en "Second Opinion" (3x07, Tim Van Patten, HBO, 2001), define a Tony como un buen hombre y un buen padre de familia. De esta forma, Carmela, además, se convierte en cómplice de las estrategias de los autores de querer destacar las características favorables del antihéroe y así lograr la simpatía de los espectadores. Ahora bien, como antídoto a esta intención, los autores de inmediato la contrarrestan a través de las palabras poco complacientes del Dr. Krakower (que, como hemos dicho más arriba, forma parte de ese grupo de personajes que bajo ningún concepto aceptan la cara positiva del antihéroe), que destacan de forma muy contundente la cara más siniestra de Tony Soprano.

A diferencia de Carmela, el principal valor de la Dra. Melfi es que, aun a pesar de las tentaciones, nunca cruzará la línea. Como hemos visto más arriba, supo mantenerse en silencio pese a su merecido deseo de venganza y la insistencia de Tony de que le contase lo que le pasaba. Como también supo decir que no a las reiteradas pretensiones que este le plantea a lo largo de la serie. Y, muy especialmente, supo cerrarle definitivamente la puerta cuando ella misma se convenció de que la terapia no solo no era beneficiosa, sino que tenía el efecto contrario: alimentaba su naturaleza psicópata. Ejemplos todos ellos de cómo David Chase y su equipo creativo plantean a los espectadores dilemas morales a través del personaje que quizá mejor los represente en el texto. Diferentes teóricos abundan en esta idea: para Vaage, la Dra. Melfi ofrece una "metaperspectiva" de la relación del espectador con Tony (2013, p. 223; 2016, p. 53); Plourde la considera "figura intermediaria” entre ambos (2006, p. 70); y Polan sugiere que ella es un recurso con el que la narrativa juega de forma autoconsciente con los espectadores (2009, p. 84).

En cualquier caso, aunque la Dra. Melfi, como hemos analizado, no es el único personaje que no pertenece al mundo protagonizado por Tony Soprano, como tampoco lo son los espectadores, sí es el único que a través de la terapia mantendrá una larga y estrecha relación con él. Esto le permi- 
tirá conocerlo mejor, como también lo harán los espectadores. Por lo que, al igual que la audiencia, tendrá que lidiar con la complejidad del antihéroe acercándose a él cuando su cara más simpática prevalezca y, por el contrario, alejándose cuando lo haga su rostro más antipático. Igualmente, en ambos casos - en el de la Dra. Melfi porque finalmente le cierra la puerta a Tony, y en el de los espectadores porque se encuentran fuera de la ficción- no sufrirán, a diferencia del resto de los personajes centrales, las trágicas consecuencias de verse envueltos en la vida del antihéroe. Así, la Dra. Melfi puede ser considerada un recurso narrativo en manos de los autores, cuya función es la de guiar las respuestas de los espectadores a los numerosos aprietos morales que se les plantean a lo largo de su extensa relación con nuestro particular antihéroe. ${ }^{5}$

\section{Breaking Bad: secreto, sorpresa y redención}

$\mathrm{Al}$ igual que con Los Soprano, la serie de Vince Gilligan despliega una colmena de personajes que enriquecen - en unas ocasiones dificultando, en otras facilitando - la identificación emocional del espectador con el protagonista. Esta identificación ha sido teorizada, principalmente, por Murray Smith en su structure of sympathy, donde el académico británico - partiendo de presupuestos cognitivistas - desarrolla los conceptos de alineamiento (alignment) y lealtad (allegiance).

En primer lugar, explica Smith, el alignment "concerns the way a film gives us access to the actions, thoughts, and feeling of characters" [se refiere al modo en que una película nos da acceso a las acciones, los pensamientos y los sentimientos de los personajes] (Smith, 1995, p. 6). En consecuencia, nos alineamos con un personaje mediante una "relación espacio-temporal" (esto es, el relato nos muestra qué hace el personaje en su entorno) y un "acceso subjetivo" (el relato también nos revela qué siente, desea y quiere el personaje). A excepción de historias más corales como The Wire (David Simon, Ed Burns, HBO, 2002-08) o Deadwood (David Milch, HBO, 2004-2006), las dos series que nos ocupan exhiben un protagonista claro, al que seguimos con el que nos alineamos - tanto en su esfera doméstica como en el ámbito

5 En la presentación que Canet hizo en el congreso de la Society for Cognitive Studies of the Moving Image celebrado en la Universidad de Cornell (Ithaca, NY, 2016), definió a la Dra. Melfi como "meta-viewer", debido a esta funcionalidad. 
profesional. La allegiance, por su parte, "concerns the way a film attempts to marshal our sympathies for or against the various characters in the world of fiction" [se refiere al modo en que una película intenta dirigir nuestras simpatías a favor o en contra de varios personajes en el mundo de ficción] (Smith, 1995, p. 6). En este segundo proceso, el personaje se gana la aprobación del espectador. Es decir, esta complicidad, como profundiza Plantinga,

is rooted in the spectator's evaluation of the moral traits of a character. If a character is held to have morally desirable traits, the spectator will be led to sympathize with the character. Such sympathies in turn partly determine the emotional responses of spectators to the narrative situations of the film. [está enraizada en la evaluación que el espectador hace de los rasgos morales de un personaje. Si un personaje ostenta rasgos moralmente deseables, el espectador será inducido a simpatizar con el personaje. Dichas simpatías, una a una, determinan en parte las respuestas emocionales de los espectadores hacia las situaciones narrativas de una película] (2010, p. 37).

Esto no quiere decir, como es lógico, que nuestra allegiance resulte incondicional. Nuestra capacidad de simpatía hacia estos personajes no es ilimitada y esta "proactitud" se puede combinar con el desprecio hacia puntuales acciones inmorales o violentas. $\mathrm{O}$, incluso, como analizaremos más adelante, voltearse de manera radical.

En este aspecto, una de las características que definen el relato serial antiheroico es que cuestiona, con una finalidad dramática, nuestra allegiance hacia el antihéroe como una estrategia que hace avanzar la trama, generar suspenso y renovar una y otra vez los conflictos dramáticos. Los autores han de reavivar cíclicamente la simpatía que los espectadores sienten hacia el antihéroe - a pesar de sus inmoralidades - para que los conflictos se multipliquen y la historia pueda dilatarse durante varias temporadas. Y lo hacen a través de las relaciones con los otros personajes relevantes de la serie, como estamos viendo. Esta es la novedad que proponemos en este artículo.

De hecho, en Breaking Bad, a diferencia de los espectadores (que tienen un conocimiento $\operatorname{casi}^{6}$ omnisciente de Walter White), la gran mayoría

6 En general, Breaking Bad adopta una focalización principal — por emplear la terminología genettiana- a través del personaje de Walter White. Sin embargo, resulta sintomático, como veremos más adelante, cómo el final de la 
de los personajes desconocen su doble faceta y, por tanto, son ajenos a la nihilista conversión de "Mr. Chips en Scarface." Durante la inmensa mayoría del relato, familiares como Walter Jr, Hank o Marie simplemente ven a un padre con cáncer o a un cuñado de vida aburrida, inmerso en la crisis de los cincuenta. Este velo provoca en el espectador un efecto balsámico, puesto que cualquier desmán primero, crimen después, de Walter White siempre contará con una escena posterior en la que, gracias a las interacciones con sus familiares, nos reconciliamos con Walter. Esto lo podemos definir como re-allegiance, ya que su lado positivo de nuevo será subrayado. Como hemos explicado en otro sitio, es precisamente la presencia de la familia la que sirve de contrapeso a las detestables acciones, de modo que una y otra vez el relato nos recuerda que las acciones del profesor de Química cuentan con el atenuante del legado económico que dejar a sus vástagos cuando el cáncer lo devore.

Incluso, hay ocasiones en las que esa falta de conocimiento de la villanía de Walter - por parte de sus allegados — sirve para ofrecer al propio protagonista una salida emocional, un entorno en el que despresurizar su tensión violenta. Así ocurre, por citar un ejemplo señero, en "Salud" (4x10, Michelle MacLaren, AMC, 2011), cuando un decaído Walter se sincera con su hijo, en una genuina petición de perdón y auxilio. Escenas como esa permiten que Walter muestre una vulnerabilidad que no se consiente en otros entornos de su vida que habilita de nuevo esa victimización que tanto influye en la evaluación moral por parte del espectador. Es decir, el hecho de que muchos personajes detecten en Walter debilidad (como hace Hank) y le muestren afecto incondicional (como hace Walter Jr) influyen decisivamente en la ecuación emocional y moral del antihéroe y así condicionar la simpatía del espectador hacia ellos.

En general, en el equilibrio dramático de la serie, siempre resulta crucial la tensión entre verdad y apariencia. De hecho, las dos claves narrativas

cuarta temporada quiebra el punto de vista en la preparación del asesinato de Gus Fring. Por un lado, la razón está en aumentar la sorpresa; pero, por otro, hay un exitoso intento por ocultarnos una acción moralmente muy discutible - el envenenamiento de Brock - y desvelarla solo cuando conocemos el éxito de la operación.

7 Así es como Vince Gilligan definió la transformación de su protagonista en el primer pitch que tuvo que hacer para que su proyecto viese la luz. 
de la última temporada tienen que ver con las consecuencias del reconocimiento de esa cara B de Walter. Por un lado, como puente entre "Gliding Over All” (5x08, Michelle MacLaren, AMC, 2012) y "Blood Money" (5x09, Bryan Cranston, AMC, 2013), tenemos a Hank reordenando todas las piezas para desenmascarar la identidad de Heisenberg. Su enfrentamiento en el garaje en "Blood Money" ("It was you! Heisenberg!" [iEras tú! ¡Heisenberg!], "Maybe your best course would be to tread lightly" [Puede que tu mejor opción sea la de andarte con cuidado] acelera la trama, desde el punto de vista narrativo, hasta su fatal desenlace. Sin embargo, desde el punto de vista de la structure of sympathy del relato, este decisivo reconocimiento de la verdad oculta, no "condena" a Walter al terreno de la antipatía del espectador. No pretendemos caer en las afirmaciones tajantes, más aún conscientes de la complejidad moral de Breaking Bad, pero sí argumentamos que, aunque Hank ostente la autoridad moral en este punto del relato, el juego del gato y el ratón que se despliega desde "Blood Money" hasta "Ozymandias" (5x14, Rian Johnson, AMC, 2013), cuando Hank, finalmente, muere asesinado a manos de Todd y su banda, provoca un reagrupamiento del espectador en torno a Walter. Por supuesto que la audiencia entiende las razones de Hank y es consciente de que la legalidad y el sentido de justicia le asisten. Sin embargo, hay varios elementos que impiden igualmente el desprecio hacia Walter: en primer lugar, sus esfuerzos — con el cáncer ya de vuelta - por salvar a su familia de la cárcel y la deshonra. En segundo lugar, la explicitación de un código moral básico con el que la audiencia se siente identificada, esto es, su insistencia en no dañar a Hank bajo ningún concepto. Y, en tercer lugar, como ampliaremos más abajo, el apoyo incondicional de Skyler, el personaje que hasta este momento del relato más había podido hacer tambalear la "proactitud" hacia Mr White.

No obstante, los dos primeros de los tres contrapesos recién citados se desmoronan en el otro reconocimiento capital de la última temporada, el de Walter Jr en "Ozymandias", tras saber que las manos de su padre están manchadas por la muerte de su tío Hank, no duda lo más mínimo en rechazar tajante a su padre, sin posibilidades de reconciliación. Ahí es cuando, por fin, ante una línea roja que no debía haberse cruzado (la muerte de Hank), Skyler decide quebrar el apoyo incondicional a su marido y así derruir el 
último de los tres pilares que sostenían la identificación moral con nuestro protagonista. Esta absoluta caída en desgracia de Walter, sin embargo, será remontada por el relato a lo largo de sus dos últimos episodios, en los que su necesaria redención moral constituirá el tema central.

Como apuntábamos al hablar de Hank, la relación más compleja es la que protagonizan Walter y Skyler. A diferencia de su hijo y cuñado, Skyler problematiza la complicada identificación moral del espectador con el protagonista de Breaking Bad mucho antes. Así, mientras que esta descubre el secreto de Walter cuando aún sus manos no están tan manchadas de sangre ni su ego tan fuera de control, Walter Jr y Hank lo descubren, como ya hemos dicho, en las postrimerías del relato, cuando Walter ya se ha convertido en el Scarface del logline. El heroico ascenso de Walter White, es decir, su capacidad para ir subiendo de escalafón en el mundo de la droga venciendo obstáculos y derribando villanos, se topa una y otra vez con el tapón que supone Skyler. De hecho, el personaje de Skyler podría analizarse como una metarreflexión sobre los mecanismos de identificación moral en la ficción audiovisual. Inicialmente, Skyler es una rémora por su desconocimiento, puesto que no cesa de presentarse como censora de las actitudes de su marido y sospechosa de sus verdaderas intenciones. Se muestra incapaz de empatizar con Walter, a diferencia de nosotros, los espectadores, que contamos con una visión de sus acciones mucho más detallada y matizada. Por ponerlo de nuevo en términos de Murray Smith, Skyler carece de alignment hacia Walter, no tiene acceso a su interior, a sus motivaciones, a sus justificaciones. Y, por tanto, la allegiance se antoja imposible. Esto contrasta con nuestro apoyo, lealtad incondicional por Walter. La contradicción dramática — y de ahí el odio hacia Skyler de muchos espectadores ${ }^{8}$ - proviene de que se trata de su esposa y, por tanto, no es un obstáculo tan fácilmente "eliminable".

Sin embargo, conforme evoluciona el relato, en la tercera temporada, Walter se ve obligado a contarle la verdad a su mujer. Y aquí es donde el co-

8 Se ha convertido en un lugar común (Gunn, 2013) afirmar que el desprecio de buena parte del público hacia la esposa del protagonista tenía que ver con prejuicios machistas o porosas apelaciones al patriarcado, cuando realmente se trata de una calculada operación dramática. 
nocimiento de la inmoralidad de Walter acaba obteniendo la complicidad de Skyler. El acceso — aunque no sea total — al cómo y al porqué del Walter mafioso invitan a Skyler a cruzar la línea y se convierte en encubridora de sus crímenes, justificándolos con las mismas coartadas morales: el bien de la familia y el orgullo, esto es, la vergüenza de que descubran su secreto familiar. En esto las similitudes entre Skyler White y Carmela Soprano son evidentes. Una y otra vez Skyler se enfada, se sorprende, se rebela contra esa complicidad e, incluso, duda con escapar, como en "Cornered" (4x06, Michael Slovis, AMC, 2011), pero se engaña a sí misma y al final es un personaje que, gracias al conocimiento, apuesta por la victoria de su marido y las consecuencias positivas de ello. No en vano, en la clausura de la cuarta temporada, en "Face Off”, tras la liquidación de Gus Fring, Walter remarca la implicación total de su esposa al espetarle un emocionado "We won" [Ganamos] (4x13, Vince Gilligan, AMC, 2011).

La relación entre Skyler y Walter en la primera parte de la quinta temporada — donde un cada vez más amoral Walter acaba maltratándola psicológicamente - sirve para cuestionar la "proactitud" del espectador hacia Walter. El miedo de Skyler, su sumisión y su incomodidad con la senda sanguinaria de su marido lo lleva a una suerte de intento de suicidio en "FitftyOne" (5x04, Rian Johnson, AMC, 2012), cuando se sumerge vestida en la piscina. Es decir, Skyler, junto con el Jesse que examinaremos más adelante, es el personaje clave para apuntalar, desde dentro del relato, nuestra relación emocional con Walter White. Por eso, parte de la reconciliación moral y heroica del espectador con Walter — ubicada astutamente en los últimos ocho capítulos de la quinta temporada- corre pareja a la reconciliación del matrimonio White, unido ante la adversidad que suponen las pesquisas de Hank Schrader analizadas más arriba.

Si la relación con Skyler ejerce de resistencia dramática — molesta, incluso, para muchos espectadores que la ven como un obstáculo insalvable para el éxito del protagonista — la relación entre Walter White y Jesse Pinkman ofrece justo lo contrario. Pinkman es un escudero fiel que, en el inicio del relato, estimula y apoya sin fisuras la transformación de Walter White, esto es, su éxito como narcotraficante. Es decir, el entusiasmo y la 
cooperación de Jesse resultan claves para apuntalar en el espectador la simpatía por Walt. Jesse constituye el trampolín del éxito de Walter.

Ahora bien, como le ocurre al Christopher de Los Soprano, Jesse querrá también cambiar de vida - sin éxito - al constatar la amoralidad en la que cada vez está más inmerso. Por eso, la relación entre esta variante de profesor y alumno también puede entenderse desde una lectura metatextual. Su amistad-contrato está atravesado por constantes alejamientos y reconciliaciones, en los que una y otra vez se mezclan las esferas personales y las privadas, como ocurre en toda la serie. Es decir, la relación entre Jesse y Walter es un espejo de las relaciones zigzagueantes que el espectador mantiene con el Mr. White. Jesse cuestiona su complicidad, al igual que los espectadores, pero siempre acaba regresando junto a Mr. White, también como hace la audiencia. Incluso ante algunas acciones detestables de Walter que afectan a Jesse directamente (como la muerte de Jane, el asesinato de Gale o el envenenamiento de Brock), el relato logra reconstituir la complicidad entre ambos personajes. Es decir, al igual que el relato testa los límites del espectador, y aplica una constante re-allegiance para mantenernos en el "equipo Walt”, la relación entre Walter y Jesse también atraviesa la misma dinámica.

Siguiendo este paralelismo, las manipulaciones de Walter sobre Jesse operarían de la misma forma que las manipulaciones del relato sobre nosotros: existe una structure of sympathy que nos empuja tanto a Jesse como a nosotros. ¿Cuándo se rompe? De nuevo, en paralelo con Skyler, durante la primera tanda de episodios de la quinta temporada. Ahí es cuando los imperdonables actos de Walter — el asesinato del niño de la moto y su "negocio del imperio"- dejan de remontarse mediante una explícita re-allegiance. Por ello, no es casualidad que los ocho últimos capítulos de la serie ubiquen a Jesse como antagonista de Walter. Y tampoco es casualidad que ni Walter ni nosotros, los espectadores, nos reconciliemos moralmente con el protagonista hasta que no obtiene, en el último cruce de miradas de la serie, en "Felina” (5x16, Vince Gilligan, AMC, 2013), la absolución de Jesse Pinkman.

De hecho, el cierre de Breaking Bad — a través de las relaciones de Walter con su familia y con Jesse- despliega la caída del antihéroe como 
planteamiento moralizante y propone su redención como estrategia final para la reconciliación con el espectador. Así, antes de morir contemplando con una sonrisa el laboratorio donde su "obra de arte" — la metanfetamina azul — seguía produciéndose, el fugitivo Walter White acude a despedirse de Skyler y sus hijos, reconociendo su verdadera motivación, el orgullo (Echart y García, 2014, pp. 83-84), como parte de su arrepentimiento: "I did it for me. I liked it. I was good at it and I was really... I was alive" [Lo hice por mí. Me gustaba. Era bueno en ello y me sentía realmente... me sentía vivo]. Así pues, es necesaria su petición de perdón como primer paso para que la lealtad del espectador hacia él, si en algún momento se perdió, se vuelva a recuperar.

De este modo, la serie se cierra con la redención del protagonista, pero con la lectura moralizante que la narración quiere ofrecerles a sus espectadores: finalmente, el antihéroe tiene que sufrir las consecuencias de sus abusos inmorales; por lo que el final resulta un perverso happy end en el que su protagonista debe morir por imperativo de una sentencia ejemplificante. Aun así, hubo quien criticó el final precisamente por resultar demasiado complaciente al no hacerle pagar a Walter más caro sus despotismos (Nussbaum, 2013). De esta forma, podemos pensar que puede haber espectadores que no quisieran reconciliarse con Walter y que, por tanto, no le hubiesen dado la oportunidad de su redención final mediante la restitución del caos causado a su familia.

\section{Conclusión}

Esta ambivalente recepción en torno al final de Breaking Bad sintetiza a la perfección la compleja moralidad del antihéroe televisivo. En este artículo, hemos intentado analizarla a través de la relación que Walter White y Tony Soprano, como ejemplos relevantes de esta figura, mantienen con otros personajes a lo largo de la serie. Esos personajes, al igual que los espectadores, tienen que lidiar con la contradicción moral de los protagonistas, por lo que se convierten en vehículos privilegiados en manos de los guionistas para provocar y guiar el abanico posible de respuestas emocionales y morales de los espectadores en su relación con el antihéroe. En este aspecto, reside la novedad de este artículo, en el que hemos propuesto escrutar cómo la 
relación entre el antihéroe y el resto de los personajes puede ampliar, modificar y desequilibrar las respuestas emocionales de los espectadores a lo largo de la serialidad antiheroica.

Como hemos visto, en ambas narrativas, se despliegan personajes que desempeñan roles semejantes en el desarrollo de la narrativa del antihéroe y que cumplen un papel central en la relativización de la ambigüedad moral del antihéroe. Carmela Soprano y Skyler White, por ejemplo, prolongan la complicidad con los desmanes de los protagonistas al mismo tiempo que provocan una cíclica tensión en torno a los límites de su propia moralidad. Christopher Moltisanti y Jesse Pinkman son ambos víctimas de las redes perversas que extiende Tony Soprano y Heisenberg en sus ambiciones egoístas. Asimismo, en ambas narrativas, se hace uso del ámbito familiar como bálsamo en las relaciones del espectador con el antihéroe, ya que, a través de esta esfera, la narración muestra la vertiente más afable del antihéroe. Las relaciones establecidas en el núcleo doméstico sirven para contrarrestar las perversidades cometidas en el ámbito "profesional” y así desarrollar una espiral continua de acercamiento y alejamiento hacia el antihéroe como estrategia clave en la experiencia del espectador con este tipo de propuestas seriales. Propuesta narrativa que se lleva a cabo principalmente, como hemos querido demostrar en este artículo, a través de las relaciones que el antihéroe mantiene con otros personajes relevantes del proyecto serial.

\section{Referencias}

Beale, J. (2014). "The Strong, Silent Type": Tony Soprano, Don Draper, and the Construction of the White Male Antihero in Contemporary Television Drama (Tesis doctoral, Bowling Green State University, Ohio).

Carroll, N. (2004). Sympathy for the Devil. En R. Green y P. Vernezze (eds.), The Sopranos and philosophy: I Kill Therefore I am (pp. 121136). Chicago: Open Court Publishing.

Echart, P. y García, A. N. (2014). "Round the decay of that colossal wreck": Pride and guilt as narrative emotions in 'Breaking Bad'. En J. Blev- 
ins y D. Wood (eds.), The methods of breaking bad: Essays on narrative, character and ethics (pp. 78-93). Jefferson: McFarland.

Frye, N. (2006). Educated imagination and other writings on critical theory, 1933-1962. Toronto: University of Toronto Press.

García, A. N. (2016). Moral emotions, antiheroes and the limits of allegiance. En A. N. García (ed.), Emotions in contemporary TV series (pp. 52-70). Basingstoke: Palgrave Macmillan.

Gunn, A. (23 agosto 2013). I have a character issue. En The New York Times. Recuperado de: http://www.nytimes.com/2013/08/24/ opinion/i-have-a-character-issue.html

Hernández, E. (2013). Teasing the audience: desconfíe de Vince Gilligan. L'Atalante. Revista de Estudios Cinematográficos, 15, 42-49.

La Berge, L. C. (2015). Fiction is liquid: States of money in The Sopranos and Breaking Bad. Journal of American Studies, 49(4), 755-74.

Lavery, D. (ed.) (2006). Reading The Sopranos: Hit TV from HBO. Londres, Nueva York: IB Tauris.

Lavery, D. (2002). This thing of ours: Investigating The Sopranos. Londres: Wallflower Press.

Mittell, J. (2015). Complex TV: The poetics of contemporary television storytelling. Nueva York: New York University Press.

Nelson, R. (2016). The emergence of antiheroism in contemporary TV. En A. N. García (ed.), Emotions in contemporary TV series (pp. 5355). Basingstoke: Palgrave Macmillan.

Nochimson, M. P. (2002). “Waddaya lookin' at?”: Re-reading the gangster genre through The Sopranos. Film Quarterly, 56(2), 2-13. 
Nussbaum, E. (30 septiembre 2013). The closure-happy Breaking Bad finale. En The New Yorker. Recuperado de: http://www.newyorker. $\mathrm{com} /$ culture/culture-desk/the-closure-happy-breaking-bad-finale

Plantinga, C. (2010). "I Followed the Rules, and they all Loved You More": Moral judgment and attitudes toward fictional characters in film. Midwest Studies in Philosophy, 34(1), 34-51.

Plourde, B. (2006). Eve of destruction: Dr Melfi as Reader of The Sopranos. En D. Lavery (ed.), Reading The Sopranos: Hit TV from HBO (pp. 69-78). Londres, Nueva York: IB Tauris.

Polan, D. (2009). The Sopranos. Durham: Duke University Press.

Rush, J. (2014). 'Want to Cook?': Static and fluid layering in The Sopranos and Breaking Bad. Journal of Screenwriting, 5(3), 393-412.

Smith, M. (1995). Engaging characters: Fiction, emotion, and the cinema. Oxford: Clarendon Press.

Smith, M. (2011). Just what is it that makes tony soprano such an appealing, attractive murderer? En W. E. Jones y S. Vice (eds.), Ethics at the cinema (pp. 66-90). Oxford: Oxford University Press.

Toscano, A. A. (2014). Tony Soprano as the American Everyman and Scoundrel: How The Sopranos (re) Presents Contemporary Middle-Class Anxieties. The Journal of Popular Culture, 47(3), 451-469.

Vaage, M. B. (2013). Fictional reliefs and reality checks. Screen, 54(2), 218-37.

Vaage, M. B. (2016). The antihero in American television. Nueva York: Routledge.

Wondemaghen, M. (2015). Walter White: The psychopath to whom we can all relate? En J. Blevins y D. Wood (eds.), The methods of breaking bad: Essays on narrative, character and ethics (pp. 122-131). Jefferson: McFarland. 